\title{
aula abierta

Artículo

\section{Más allá del conocimiento. El impacto de un programa educativo universitario para mayores}

\author{
Alicia Pérez-Albéniz ${ }^{\mathrm{a}, *}$, Ana I. Pascual ${ }^{\mathrm{a}}$, M. Cruz Navarro ${ }^{\mathrm{a}}$ y Beatriz Lucas-Molinab \\ ${ }^{a}$ Universidad de La Rioja, Logroño, España \\ ${ }^{\mathrm{b}}$ Universidad de Valencia., Valencia, España
}

\section{INFORMACIÓN DEL ARTÍCULO}

\section{Historia del artículo:}

Recibido el 21 de febrero de 2014

Aceptado el 4 de junio de 2014

On-line el 30 de octubre de 2014

\section{Palabras clave:}

Programas universitarios para mayores

Envejecimiento activo

Integración social

Bienestar

Calidad de vida

\begin{abstract}
R E S U M E N
El objetivo del presente estudio fue analizar el impacto percibido por parte de los alumnos como resultado de su participación en el programa de la Universidad de la Experiencia de la Universidad de La Rioja. La muestra estuvo formada por 83 alumnos de dicho programa. Al grupo de estudiantes se les aplicó un cuestionario diseñado ad hoc para evaluar potenciales resultados a diferentes niveles: general, educativo, social, afectivo, cognitivo, físico y afectividad negativa. Los resultados obtenidos muestran que el impacto del programa trasciende la adquisición de conocimientos, alcanzando otras esferas relevantes de la persona como son la social, la psicológica y la física. Además, los resultados mostraron que las mujeres en general perciben mejores resultados a casi todos los niveles. El estudio aportó evidencia empírica a favor de tres hipótesis que vienen aceptándose desde hace años en el ámbito de la educación y de la sociología: las ventajas de la educación a lo largo de toda la vida, el hecho de que los beneficios de la educación van más allá de la adquisición de conocimientos y la importancia de la actividad física y mental en la calidad de vida de los mayores.
\end{abstract}

(c) 2014 Instituto de Ciencias de la Educación de la Universidad de Oviedo. Publicado por Elsevier España, S.L.U. Todos los derechos reservados

\section{Beyond knowledge. The impact of an university education program for older people}

A B S T R A C T

The aim of the present study was to analyze the perceived impact of the University of Experience program at the University of La Rioja on students. The study sample consisted of 83 students from this education program for older people. The group of students was administered a questionnaire designed ad hoc to assess potential outcomes at different levels: general, educational, social, affective, cognitive, physical and negative affectivity. The results show that the impact of the program goes beyond the acquisition of knowledge, reaching other relevant aspects of personal functioning such as the social, the psychological, and the physical areas. In addition, the results showed that women generally perceive better results at almost all levels. The study provided empirical evidence for three hypotheses that have been accepted in the field of education and sociology for years: the advantages of education throughout life, the fact that the benefits of education go beyond the acquisition of knowledge, and the importance of physical and mental activity on the quality of life of older people.

(c) 2014 Instituto de Ciencias de la Educación de la Universidad de Oviedo. Published by Elsevier España, S.L.U. All rights reserved.
* Autor para correspondencia. Departamento de Ciencias de la Educación, Universidad de La Rioja, C/Luis de Ulloa, s/n (Edificio Vives), 26004 Logroño, España.

Correo electrónico: alicia.perez@unirioja.es (A. Pérez-Albéniz).

\section{Introducción}

A partir de los años 90, adquiere una gran popularidad en España la idea del envejecimiento activo (active ageing). La Organización Mundial de la Salud define este término como el proceso de aprovechar al máximo las oportunidades para tener 
un bienestar físico, psíquico y social durante toda la vida (OMS, 2002). El objetivo, por tanto, es extender la calidad de vida a las personas de edad avanzada. El término activo no se refiere solo a la actividad física, sino que anima a seguir participando en los acontecimientos sociales, económicos, culturales, espirituales y cívicos. Según los principios de Naciones Unidas, los 3 pilares que garantizan el envejecimiento activo son: salud, participación en la sociedad y seguridad (Ministerio de Sanidad, 2010).

Es precisamente en el marco del segundo de estos pilares, la participación en la sociedad, en la que han aparecido en los últimos años los programas universitarios para mayores. Estas aulas tienen como objetivo potenciar la integración de las personas mayores en el contexto sociocultural que representa la Universidad, favoreciendo su calidad de vida a través del ejercicio intelectual. Los programas educativos para mayores pueden ser enmarcados, además, en los objetivos relacionados con la educación a lo largo de toda la vida que, de manera creciente, se van incorporando a las agendas políticas de instituciones y gobiernos, de ámbito nacional y supranacional. Sirvan de ejemplo las referencias a este objetivo en la Unión Europea, en el Ministerio de Educación español, en las leyes educativas aprobadas en los últimos años e, incluso, en organizaciones específicas, como es el caso de la Organización Internacional del Trabajo (Comisión Europea, 2010; MECU, 2013; Organización Internacional del Trabajo, 2005). Desde la Unión Europea se viene realizando desde el año 2000 una apuesta clara por la educación a lo largo de toda la vida y se vincula este objetivo a metas relacionadas con el progreso económico, el desarrollo personal y la igualdad de oportunidades, entre otras.

En 2005, la Universidad de La Rioja decide sumarse a diversas universidades españolas (véase Orte, Ballester y Touza, 2004) en el propósito de poner en marcha un proyecto educativo dirigido a la población adulta que pretende promover su incorporación a la vida universitaria a través de programas de formación paralelos a las enseñanzas regladas.

La oferta educativa en esta universidad nace comprometida con unos objetivos comunes en muchos de los programas vigentes en otras universidades. El planteamiento de objetivos es compartido en la esfera de lo educativo y cultural, pero desde una perspectiva mucho más ambiciosa: conseguir mejorar la calidad de vida de las personas que se implicaran en el mismo a diferentes niveles. En este sentido, los programas pretenden responder a las demandas de formación de los adultos en el ámbito universitario, además de contribuir a su adaptación a la sociedad, impulsar sus capacidades cognitivas y emocionales, y favorecer su desarrollo personal y su máxima integración social.

Desde hace algunos años, existe un interés general por evaluar la calidad de los programas universitarios para mayores en España ( $\mathrm{p}$. ej., Palmero y Eguizábal, 2008). Sin embargo, son escasos los estudios que se han llevado a cabo. Existen algunos ejemplos de estudios que intentan analizar la satisfacción de los alumnos. Por ejemplo, Navarro y Pérez de Albéniz (2010) pusieron de manifiesto que existía un grado de satisfacción muy elevado con el programa, las materias y los profesores que las imparten. En la misma línea, el estudio de Rubio Herrera (2000) indicaba unos altos índices de satisfacción tanto de los mayores que participan, como de profesores y coordinadores. $\mathrm{O}$ uno más reciente, realizado en la Universidad de Granada, que mostraba que, independientemente de la edad y el género de la persona, el participar en el Aula de Mayores estaba relacionado con una alta satisfacción vital (Castellón, Gómez y Martos, 2004).

Sin embargo, cabe preguntarse qué se llevan los alumnos de su paso por el programa de la Universidad de la Experiencia de la Universidad La Rioja y si los objetivos mencionados son alcanzados. Son numerosos los comentarios que estos alumnos trasladan a quienes vienen gestionando el programa sobre los beneficios obtenidos al participar en el mismo. Sin embargo, es necesaria una evaluación más rigurosa en la que los resultados obtenidos sean aportados por el mayor número de alumnos, de los que hayan acabado el programa o que estén a punto de hacerlo. Se requiere conocer si los alumnos del programa perciben haber conseguido resultados negativos y positivos en las áreas de educativa, social, afectiva, cognitiva o física. Con esta intención, se llevó a cabo el presente estudio.

Asimismo, es interesante conocer si variables como el género, la edad, la situación laboral o los estudios previos a su inclusión en el programa hacen que unos grupos de alumnos perciban mejores resultados que otros de su paso por la Universidad de la Experiencia.

\section{Método}

\section{Participantes}

La muestra estuvo compuesta por un total de 83 alumnos de la Universidad de la Experiencia de la Universidad de La Rioja. Del total, 33 estudiantes pertenecían al grupo de los cursos de formación permanente (para los alumnos que han superado los estudios de la Universidad de la Experiencia) de la sede de Logroño, 22 pertenecían al grupo de los cursos de formación permanente de la sede de Calahorra y 28 alumnos pertenecían al grupo del Módulo 3 del programa educativo de Logroño (último curso de los estudios de la Universidad de la Experiencia). Los grupos de alumnos que respondieron al cuestionario representaban un alto porcentaje de los alumnos matriculados en cada grupo (el 55,0, el 73,3 y el 87,5\%, respectivamente).

En cuanto al género, 25 de los alumnos que respondieron el cuestionario eran hombres y 56 eran mujeres. Dos de los participantes no informaron sobre su género. La media de edad de los participantes fue de 62,57 $(D T=6,50)$. El rango de edad osciló entre los 48 y los 79 años.

Ochenta y un participantes aportaron información acerca de su situación laboral, que se distribuía de la siguiente manera: el 13,6\% estaba trabajando, el 9,9\% se encontraba desempleado, el 66,7\% era jubilado y el 9,9\% se encontraba en otras situaciones laborales (p. ej., realizando trabajos del hogar).

Respecto a los estudios que poseían con anterioridad a su implicación en la Universidad de la Experiencia, el 2,4\% no tenía estudios, el 20,5\% había cursado estudios primarios, el 48,2\% tenía el título de Bachiller o Formación Profesional y, finalmente, el 28,9\% había cursado estudios universitarios.

\section{Instrumentos}

\section{Cuestionario ad hoc para la valoración del impacto de la}

Universidad de la Experiencia en los alumnos

El procedimiento para la elaboración del cuestionario ad hoc se llevó a cabo en 2 fases. En la primera, se solicitó a un grupo de exalumnos que describieran cuáles eran las áreas en las que creían que la participación en la Universidad de la Experiencia tenía un impacto relevante. En la segunda fase, se realizó una elaboración y una categorización de estas respuestas, quedando finalmente un total de 35 ítems que se agrupaban en función de su contenido en 7 factores teóricos. Se denominó factor general al ítem 1 por no ajustar conceptualmente con ninguna de las dimensiones restantes. En segundo lugar, se denominó factor educativo al factor compuesto por los ítems 2, 3, 4, 5, 6 y 7. En tercer lugar, se denominó factor social al compuesto por los ítems 8, 9, 10, 11 y 12. En cuarto lugar, se construyó el factor afectivo, el compuesto por los ítems 13, 14, $15,16,19,20,21,22,24,25$ y 26. Asimismo, se denominó factor cognitivo el compuesto por los ítems 17,18 y 23 . El factor físico estuvo compuesto por los ítems 27 y 28 . Un último factor fue denominado afectividad negativa e incluía posibles efectos negativos a nivel afectivo (estrés, frustración, ansiedad, entre otros) percibidos por el paso por el programa de la Universidad de la Experiencia y estuvo compuesto por los ítems 29, 30, 31, 32, 33, 34 y 35. El 
Tabla 1

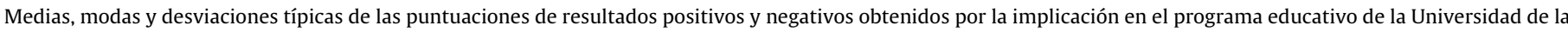
Experiencia

\begin{tabular}{|c|c|c|c|}
\hline & Moda & Media & Desviación típica \\
\hline \multicolumn{4}{|l|}{ Aspectos positivos como resultado de la participación en el programa: } \\
\hline \multicolumn{4}{|l|}{ General } \\
\hline 1. He obtenido satisfacción personal por realizar actividades que me enriquecen & 5 & 4,43 & 0,58 \\
\hline \multicolumn{4}{|l|}{ Factor educativo-cultural } \\
\hline $\begin{array}{l}\text { 2. Me ha gustado poder realizar estudios que no había podido cursar anteriormente o recuperar y } \\
\text { enriquecer los que ya tenía }\end{array}$ & 5 & 4,34 & 0,75 \\
\hline 3. He experimentado una mejora en mi cultura general & 4 & 3,89 & 0,82 \\
\hline 4. He superado un reto a nivel intelectual & 4 & 3,59 & 0,96 \\
\hline $\begin{array}{l}\text { 5. He tenido la oportunidad de acercarme a materias desconocidas y a otras conocidas desde otros } \\
\text { puntos de vista }\end{array}$ & 5 & 4,21 & 0,80 \\
\hline $\begin{array}{l}\text { 6. Me siento motivado para profundizar en algún área de conocimiento de manera autónoma una vez } \\
\text { que he cursado algunas asignaturas }\end{array}$ & 4 & 3,73 & 0,96 \\
\hline $\begin{array}{l}\text { 7. He seguido desarrollando habilidades adquiridas a lo largo de la vida que corrían el riesgo de oxidarse } \\
\text { prematuramente }\end{array}$ & 4 & 3,70 & 1,08 \\
\hline \multicolumn{4}{|l|}{ Factor social } \\
\hline 8. He ganado relaciones de amistad & 5 & 4,10 & 0,97 \\
\hline 9. He profundizado más en las relaciones que he establecido & 4 & 3,49 & 1,05 \\
\hline $\begin{array}{l}\text { 10. Las actividades que han surgido «alrededor» del proyecto (salidas, comidas, cumpleaños, etc.) me } \\
\text { han enriquecido personalmente }\end{array}$ & 4 & 3,49 & 1,02 \\
\hline 11. Ahora puedo hablar con personas con las que antes no me atrevía & 3 & 2,72 & 1,30 \\
\hline 12. Me siento integrado en la Universidad & 5 & 4,10 & 0,94 \\
\hline \multicolumn{4}{|l|}{ Factor psicológico: afectivo y cognitivo } \\
\hline 13. Ha aumentado mi autoestima porque superar el programa era todo un reto personal para mí & 4 & 3,59 & 1,15 \\
\hline $\begin{array}{l}\text { 14. Me ha aportado bienestar psicológico general (me siento renovado, más vivo, en rodaje continuo, } \\
\text { etc.) }\end{array}$ & 4 & 3,90 & 1,01 \\
\hline 15. He reducido sentimientos de ansiedad o depresión previos & 1 & 2,52 & 1,38 \\
\hline $\begin{array}{l}\text { 16. Participar en este programa me ha ayudado a superar alguna situación vital traumática (viudedad, } \\
\text { enfermedad o pérdida de algún familiar, amigo o similar) }\end{array}$ & 1 & 2,36 & 1,51 \\
\hline 17. Mi capacidad intelectual ha mejorado respecto a la que tenía antes & 3 & 3,40 & 0,98 \\
\hline 18. Ha mejorado mi memoria & 4 & 3,11 & 1,15 \\
\hline 19. Ha sido una alternativa enriquecedora para hacer frente a la jubilación & 5 & 3,58 & 1,36 \\
\hline 20. Me ha ayudado a sentir que puedo seguir enfrentando retos día a día y superarlos & 4 & 3,63 & 1,27 \\
\hline 21. He descubierto el placer de compartir con los demás lo que uno sabe & 4 & 3,66 & 1,05 \\
\hline 22. He conseguido vencer temores y complejos & 3 & 2,81 & 1,32 \\
\hline 23. Ahora soy más receptivo a aprender a usar el móvil (agenda, mensajes, etc.) y el ordenador & 4 & 3,36 & 1,32 \\
\hline 24. Ha mejorado mi humor & 4 & 2,93 & 1,27 \\
\hline 25. Asistir a las clases me ha servido para liberarme de mis múltiples obligaciones y pensar más en mí & 4 & 3,18 & 1,36 \\
\hline 26. Soy más tolerante y humilde, porque acepto las observaciones de los demás y mis errores & 4 & 3,48 & 0,98 \\
\hline \multicolumn{4}{|l|}{ Factor físico } \\
\hline 27. He experimentado una mejora en mi estado físico general & 4 & 2,67 & 1,25 \\
\hline 28. Ha disminuido la frecuencia con la que voy al médico & 1 & 1,94 & 1,16 \\
\hline \multicolumn{4}{|l|}{ Afectividad negativa } \\
\hline $\begin{array}{l}\text { 29. Me he sentido culpable porque he dejado de hacer algunas actividades en familia o con amigos que } \\
\text { antes hacía }\end{array}$ & 1 & 1,45 & 0,91 \\
\hline 30. Me he sentido en algunos momentos estresado y agobiado & 2 & 2,33 & 1,12 \\
\hline 31. Me he sentido frustrado por no obtener los resultados que yo deseaba por la exigencia del programa & 1 & 1,65 & 0,81 \\
\hline 32. Me he sentido frustrado por no obtener los resultados que yo deseaba por mi falta de capacidad & 1 & 1,61 & 0,94 \\
\hline $\begin{array}{l}\text { 33. Me he sentido frustrado por no obtener los resultados que yo deseaba porque no he dispuesto del } \\
\text { tiempo suficiente }\end{array}$ & 1 & 1,55 & 0,81 \\
\hline 34. Me he sentido mal por problemas que he tenido con algunos compañeros & 1 & 1,48 & 0,98 \\
\hline 35. Me he sentido mal por problemas que he tenido con algunos profesores & 1 & 1,30 & 0,85 \\
\hline
\end{tabular}

cuestionario resultante compuesto por los 35 ítems se presentaba con una escala de respuesta en formato Likert de 5 puntos (siendo 1 ninguna intensidad y 5 mucha intensidad). Los ítems del cuestionario se muestran en las tablas 1 y 2 del apartado de resultados.

\section{Procedimiento}

La administración del cuestionario se llevó a cabo de forma colectiva en grupos de entre 10 y 30 participantes. Se informó en todo momento de la confidencialidad de las respuestas, así como del carácter voluntario de la participación. No se ofreció ningún tipo de gratificación por la participación en el estudio.

\section{Análisis de datos}

Se realizaron análisis descriptivos y se utilizaron análisis multivariados de la variancia (MANOVAS) con los diferentes factores intersujetos (género, edad, situación laboral y nivel de estudios previo) para todas las variables dependientes (factores general, educativo, social, afectivo, cognitivo y afectividad negativa). Asimismo, se realizaron análisis follow up mediante ANOVA para cada factor estudiado y se llevaron a cabo pruebas de comparación múltiple de medias Tukey.

\section{Resultados}

En la tabla 1 se presentan los efectos positivos que los alumnos percibían como más intensos debido a su participación en el programa educativo de la Universidad de la Experiencia. En concreto, los ítems con una media más alta fueron, en el siguiente orden: (1) haber obtenido satisfacción personal por realizar actividades que les enriquecían; (2) satisfacción por haber podido realizar estudios que no habían podido cursar anteriormente o recuperar 
Tabla 2

Frecuencia con la que los alumnos seleccionaron cada ítem como uno de los 3 más importantes en el cuestionario (los ítems se presentan ordenados por frecuencia)

\begin{tabular}{|c|c|}
\hline & Frecuencia \\
\hline $\begin{array}{l}\text { 1. He obtenido satisfacción personal por realizar } \\
\text { actividades que me enriquecen }\end{array}$ & 31 \\
\hline 2. He ganado relaciones de amistad & 22 \\
\hline $\begin{array}{l}\text { 3. Me ha gustado poder realizar estudios que no había } \\
\text { podido cursar anteriormente o recuperar y enriquecer } \\
\text { los que ya tenía }\end{array}$ & 19 \\
\hline $\begin{array}{l}\text { 4. He tenido la oportunidad de acercarme a materias } \\
\text { desconocidas y a otras conocidas desde otros puntos de } \\
\text { vista }\end{array}$ & 19 \\
\hline $\begin{array}{l}\text { 5. Me ha aportado bienestar psicológico general (me siento } \\
\text { renovado, más vivo, en rodaje continuo, etc.) }\end{array}$ & 14 \\
\hline 6. He experimentado una mejora en mi cultura general & 12 \\
\hline $\begin{array}{l}\text { 7. Ha aumentado mi autoestima porque superar el } \\
\text { programa era todo un reto personal para mí }\end{array}$ & 12 \\
\hline 8. Me siento integrado en la Universidad & 11 \\
\hline $\begin{array}{l}\text { 9. Ha sido una alternativa enriquecedora para hacer frente } \\
\text { a la jubilación }\end{array}$ & 11 \\
\hline $\begin{array}{l}\text { 10. Mi capacidad intelectual ha mejorado respecto a la que } \\
\text { tenía antes }\end{array}$ & 10 \\
\hline $\begin{array}{l}\text { 11. Soy más tolerante y humilde, porque acepto las } \\
\text { observaciones de los demás y mis errores }\end{array}$ & 8 \\
\hline 12. He superado un reto a nivel intelectual & 7 \\
\hline $\begin{array}{l}\text { 13. Me siento motivado para profundizar en algún área de } \\
\text { conocimiento de manera autónoma una vez que he } \\
\text { cursado algunas asignaturas }\end{array}$ & 7 \\
\hline $\begin{array}{l}\text { 14. He seguido desarrollando habilidades adquiridas a lo } \\
\text { largo de la vida que corrían el riesgo de oxidarse } \\
\text { prematuramente }\end{array}$ & 6 \\
\hline $\begin{array}{l}\text { 15. He descubierto el placer de compartir con los demás lo } \\
\text { que uno sabe }\end{array}$ & 6 \\
\hline $\begin{array}{l}\text { 16. Ahora soy más receptivo a aprender a usar el móvil } \\
\text { (agenda, mensajes, etc.) y el ordenador }\end{array}$ & 6 \\
\hline $\begin{array}{l}\text { 17. Asistir a las clases me ha servido para liberarme de mis } \\
\text { múltiples obligaciones y pensar más en mí }\end{array}$ & 6 \\
\hline $\begin{array}{l}\text { 18. Me ha ayudado a sentir que puedo seguir enfrentando } \\
\text { retos día a día y superarlos }\end{array}$ & 5 \\
\hline $\begin{array}{l}\text { 19. He experimentado una mejora en mi estado físico } \\
\text { general }\end{array}$ & 5 \\
\hline $\begin{array}{l}\text { 20. Me he sentido en algunos momentos estresado y } \\
\text { agobiado }\end{array}$ & 4 \\
\hline $\begin{array}{l}\text { 21. Las actividades que han surgido «alrededor» del } \\
\text { proyecto (salidas, comidas, cumpleaños, etc.) me han } \\
\text { enriquecido personalmente }\end{array}$ & 3 \\
\hline $\begin{array}{l}\text { 22. Participar en este programa me ha ayudado a superar } \\
\text { alguna situación vital traumática (viudedad, enfermedad } \\
\text { o pérdida de algún familiar, amigo o similar) }\end{array}$ & 3 \\
\hline $\begin{array}{l}\text { 23. Me he sentido frustrado por no obtener los resultados } \\
\text { que yo deseaba por la exigencia del programa }\end{array}$ & 3 \\
\hline $\begin{array}{l}\text { 24. He profundizado más en las relaciones que he } \\
\text { establecido }\end{array}$ & 2 \\
\hline $\begin{array}{l}\text { 25. Ahora puedo hablar con personas con las que antes no } \\
\text { me atrevía }\end{array}$ & 2 \\
\hline 26. Ha mejorado mi memoria & 2 \\
\hline 27. He conseguido vencer temores y complejos & 2 \\
\hline 28. Ha mejorado mi humor & 1 \\
\hline $\begin{array}{l}\text { 29. Me he sentido frustrado por no obtener los resultados } \\
\text { que yo deseaba por mi falta de capacidad }\end{array}$ & 1 \\
\hline $\begin{array}{l}\text { 30. Me he sentido mal por problemas que he tenido con } \\
\text { algunos compañeros }\end{array}$ & 1 \\
\hline $\begin{array}{l}\text { 31. Me he sentido mal por problemas que he tenido con } \\
\text { algunos profesores }\end{array}$ & 1 \\
\hline $\begin{array}{l}\text { 32. He reducido sentimientos de ansiedad o depresión } \\
\text { previos }\end{array}$ & 0 \\
\hline 33. Ha disminuido la frecuencia con la que voy al médico & 0 \\
\hline $\begin{array}{l}\text { 34. Me he sentido culpable porque he dejado de hacer } \\
\text { algunas actividades en familia o con amigos que antes } \\
\text { hacía }\end{array}$ & 0 \\
\hline $\begin{array}{l}\text { 35. Me he sentido frustrado por no obtener los resultados } \\
\text { que yo deseaba porque no he dispuesto del tiempo } \\
\text { suficiente }\end{array}$ & 0 \\
\hline
\end{tabular}

y enriquecer los que ya tenían; (5) haber tenido la oportunidad de acercarse a materias desconocidas y otras conocidas desde otros puntos de vista; (8) haber ganado relaciones de amistad, y (12) sentirse integrados en la Universidad. Los ítems anteriormente mencionados hacen referencia a los factores teóricos educativo y social, respectivamente.

Aunque no tan destacables, las puntuaciones medias de los ítems correspondientes al factor psicológico fueron medias o altas, indicando que muchos alumnos percibían una mejora a nivel psicológico. Las excepciones en este factor las constituyen los ítems 16 y 15 , que obtuvieron puntuaciones moderadas.

Es destacable además el ítem 19, que hacía referencia a que el programa educativo había supuesto una alternativa enriquecedora para hacer frente a la jubilación, dado que, aunque la media no haya sido de las más altas, su moda sí muestra una alta frecuencia de puntuaciones máximas. Además, es necesario tener en cuenta que no todos los alumnos son jubilados.

En cuanto al factor físico, los ítems mostraron puntuaciones bajas o moderadas. La media de las puntuaciones para la afirmación «He experimentado una mejora en mi estado físico general» fue moderada, pero de nuevo es interesante observar que la moda que muestra este ítem es de 4, indicando que, aunque no para la mayoría de los alumnos, para algunos ha sido un resultado importante.

Respecto al factor afectividad negativa (ítems 29 a 35 del cuestionario), las respuestas vienen a reforzar el panorama positivo que apuntan los resultados anteriores. De hecho, los alumnos no afirmaban haber experimentado efectos negativos. Tal y como puede observarse en la tabla 1, todos los ítems fueron evaluados con puntuaciones muy bajas. El único aspecto a señalar es que el ítem que hacía referencia a los sentimientos de estrés generados por la exigencia del programa recibió puntuaciones mayores, pero dentro del rango de las más bajas igualmente.

Respecto a los resultados a los que los alumnos conceden mayor relevancia, se les solicitó a los alumnos la selección de 3 de los resultados expuestos con el fin de conocer el grado de importancia que les concedían, independientemente de su nivel de consecución. A continuación, en la tabla 2, se expone la frecuencia total con la que cada ítem fue seleccionado.

Los alumnos destacaron la satisfacción personal por realizar actividades que les enriquecen y la oportunidad que les brinda el programa para ganar relaciones de amistad. Además, y en coherencia con los resultados que percibían haber obtenido, los alumnos puntuaron como resultados más relevantes aquellos que hacían referencia al nivel cultural y educativo (ítems 2, 3 y 19).

Por otra parte, los alumnos destacaron como relevantes algunos beneficios a nivel psicológico (mejora de la autoestima, del bienestar psicológico general, de la capacidad intelectual). Cabe destacar que, aunque los alumnos sí afirmaban que obtenían resultados moderados a este nivel (tabla 1), percibían como relevante el impacto que el programa puede tener en este aspecto.

En relación con los resultados obtenidos en el primer análisis, los alumnos destacaron moderadamente la importancia del programa como alternativa enriquecedora para hacer frente a la jubilación.

Finalmente, con el objetivo de conocer sobre qué dimensiones era mayor el impacto del programa, los 35 ítems del cuestionario fueron agrupados en función de su contenido en factores, construyéndose un total de 6 factores teóricos (cuyo contenido se ha planteado con anterioridad en el apartado de instrumentos).

Como puede observarse en la tabla 3 , la media más alta corresponde al impacto obtenido en el factor educativo $(\bar{X}=3,9)$ y la media más baja al del factor físico $(\bar{X}=2,3)$. Entre ellos aparecen los factores social $(\bar{X}=3,6)$, cognitivo $(\bar{X}=3,3)$ y afectivo $(\bar{X}=3,2)$ corroborando los resultados obtenidos cuando se han considerado cada uno de los ítems por separado.

La desviación típica obtenida es bastante amplia (desde 0,8 hasta 1 punto) en todos los factores salvo en el educativo. En general, el 
Tabla 3

Medias (desviaciones típicas) de cada factor en el total de la muestra y función del género y de la edad de los alumnos

\begin{tabular}{|c|c|c|c|c|c|}
\hline & $\begin{array}{l}\text { Hombre } \\
(n=25)\end{array}$ & $\begin{array}{l}\text { Mujer } \\
(n=56)\end{array}$ & $\begin{array}{l}\text { Menores de } 65 \text { años } \\
(n=44)\end{array}$ & $\begin{array}{l}\text { Mayores de } 65 \text { años } \\
(n=39)\end{array}$ & $\begin{array}{l}\text { Global } \\
(n=83)\end{array}$ \\
\hline Factor educativo & $3,7(0,6)$ & $4(0,6)$ & $3,9(0,6)$ & $3,9(0,6)$ & $3,9(0,6)$ \\
\hline Factor social & $3,2(0,9)$ & $3,7(0,6)$ & $3,6(0,7)$ & $3,6(0,9)$ & $3,6(0,8)$ \\
\hline Factor cognitivo & $3,1(0,9)$ & $3,4(0,9)$ & $3,3(0,9)$ & $3,3(0,9)$ & $3,3(0,9)$ \\
\hline Factor afectivo & $3(0,9)$ & $3,3(0,8)$ & $3,3(0,9)$ & $3,1(0,9)$ & $3,2(0,9)$ \\
\hline Factor físico & $2,3(1,1)$ & $2,3(1)$ & $2,3(1,1)$ & $2,4(1)$ & $2,3(1)$ \\
\hline
\end{tabular}

Tabla 4

Medias (desviaciones típicas) de cada factor en función de la situación laboral de los alumnos

\begin{tabular}{|c|c|c|c|c|c|}
\hline & $\begin{array}{l}\text { Empleados } \\
(n=11)\end{array}$ & $\begin{array}{l}\text { Desempleados } \\
(n=8)\end{array}$ & $\begin{array}{l}\text { Jubilados } \\
(n=54)\end{array}$ & $\begin{array}{l}\text { Otras } \\
(n=8)\end{array}$ & $\begin{array}{l}\text { Global } \\
(n=83)\end{array}$ \\
\hline Factor educativo & $4(0,5)$ & $4(0,5)$ & $3,9(0,6)$ & $3,6(0,7)$ & $3,9(0,6)$ \\
\hline Factor social & $3,6(0,8)$ & $3,5(0,7)$ & $3,6(0,8)$ & $3,3(1,2)$ & $3,6(0,8)$ \\
\hline Factor cognitivo & $3,5(0,7)$ & $3,3(0,7)$ & $3,3(1)$ & $2,8(1)$ & $3,3(0,9)$ \\
\hline Factor afectivo & $3,5(0,7)$ & $3,3(0,8)$ & $3,2(0,9)$ & $2,7(1,2)$ & $3,2(0,9)$ \\
\hline Factor físico & $2,4(0,9)$ & $2,3(0,8)$ & $2,3(1,1)$ & $2,2(1,1)$ & $2,3(1)$ \\
\hline
\end{tabular}

análisis de la desviación típica nos indica que esta es mayor cuanto menor es la media obtenida en los factores.

Estos factores pueden ser analizados no solo globalmente, sino agrupando a los alumnos según distintos criterios. En este caso, los criterios de agrupación que se tuvieron en cuenta fueron el género, la edad de los alumnos, la situación laboral y el nivel de estudios previos de los alumnos.

Los datos sobre el género y la edad de los alumnos se recogen asimismo en la tabla 3. El efecto principal del género no fue significativo, aunque sí mostró una tendencia a la significación (lambda de Wilk $=0,87 ; F(5,75)=2,14 ; \mathrm{p}=0,069)$. Aunque ambos grupos coincidieron en el orden de puntuación de los diferentes factores, se aprecian diferencias en cuanto al valor de dichas puntuaciones. Con la excepción del factor físico, las mujeres manifestaron haber percibido resultados más intensos a todos los niveles. Sin embargo, los ANOVA univariados indicaron únicamente un efecto significativo para el factor social $(1,79)=9,73 ; p=0,003$ y tendencial para el factor afectivo $(1,79)=2,95 ; \mathrm{p}=0,09$.

Con el fin de conocer si la edad de los alumnos influía en la valoración de los factores, la muestra fue dividida en 2 grupos (menores de 65 y mayores de 65). Los datos se recogen en la tabla 3. Como puede observarse, la percepción de los alumnos sobre los resultados obtenidos fue muy similar en ambos grupos de edad y no hubo diferencias estadísticamente significativas para esta variable (lambda de Wilk $=0,63 ; F(5,77)=0,96 ; \mathrm{p}>.05)$.

De igual manera, con el objetivo de conocer si la situación laboral tenía alguna influencia sobre la valoración que realizan los alumnos, se dividió la muestra en 4 grupos (empleados, desempleados, jubilados y otros). Los datos (tabla 4 ) no arrojaron diferencias significativas entre grupos (lambda de Wilk $=0,91 ; F(5,73)=0,42$; $\mathrm{p}>0,05$ ).

Respecto al nivel de estudios previo a su implicación en el programa de la Universidad de la Experiencia, se consideraron 4 niveles para el análisis ( sin estudios, con estudios primarios, con Bachiller o $\mathrm{FP}$, con estudios universitarios). La tabla 5 recoge las medias en cada uno de los factores evaluados. Los resultados mostraron diferencias estadísticamente significativas (lambda de Wilk Lambda =0,69; $F$ $(5,75)=1,98 ; p=0,018)$, ya que la percepción de los alumnos $\sin$ estudios era muy diferente del de los otros grupos de alumnos. No obstante, no se procederá a la interpretación de este dato por la reducida muestra en esta categoría $(n=2)$.

\section{Discusión}

De acuerdo con las hipótesis formuladas, los resultados indican que los alumnos obtienen de su participación en el programa educativo de la Universidad de la Experiencia de la Universidad de La Rioja mucho más que conocimiento. A la luz de los resultados, el impacto del programa percibido por los alumnos trasciende el ámbito educativo y alcanza todas las esferas relevantes de la persona. En este sentido, los alumnos refirieron haber obtenido también resultados positivos a nivel social y psicológico.

A nivel educativo, destacaron las siguientes percepciones por parte de los alumnos: el programa les ofrece una alta satisfacción personal por realizar actividades que les enriquecen, por haber podido realizar estudios que no habían podido cursar anteriormente o recuperar y enriquecer los que ya tenían, y valoran el haber tenido la oportunidad de acercarse a materias desconocidas y otras conocidas desde otros puntos de vista.

A nivel social, los alumnos destacaron que el programa les ha permitido establecer nuevas relaciones de amistad y el hecho de sentirse integrados en la Universidad.

Por otro lado, aunque los alumnos no destacaron los resultados obtenidos a nivel psicólogico, sí referían que el programa les ha aportado bienestar psicológico general, aumento de autoestima, mejora de la capacidad cognitiva previa y el sentimiento de que

Tabla 5

Medias (desviaciones típicas) de cada factor en función del nivel de estudios previo de los alumnos

\begin{tabular}{|c|c|c|c|c|c|}
\hline & $\begin{array}{l}\text { Sin estudios } \\
(n=2)\end{array}$ & $\begin{array}{l}\text { Primarios } \\
(n=17)\end{array}$ & $\begin{array}{l}\text { Bachiller o FP } \\
(n=40)\end{array}$ & $\begin{array}{l}\text { Universitarios } \\
(n=24)\end{array}$ & $\begin{array}{l}\text { Global } \\
(n=83)\end{array}$ \\
\hline Factor educativo & $3,9(0,6)$ & $4(0,6)$ & $3,8(0,6)$ & $4(0,6)$ & $3,9(0,6)$ \\
\hline Factor social & $4,6(0,3)$ & $3,8(0,9)$ & $3,4(0,7)$ & $3,6(0,8)$ & $3,6(0,8)$ \\
\hline Factor cognitivo & $2,9(0,7)$ & $3,7(0,7)$ & $3,3(0,9)$ & $3,1(1,1)$ & $3,3(0,9)$ \\
\hline Factor afectivo & $2,5(1,8)$ & $3,5(0,8)$ & $3,2(0,8)$ & $3,1(1)$ & $3,2(0,9)$ \\
\hline Factor físico & $2(1,4)$ & $2,4(1,2)$ & $2,3(0,9)$ & $2,3(1,1)$ & $2,3(1)$ \\
\hline
\end{tabular}


pueden seguir enfrentando retos día a día y superarlos. Asimismo, muchos destacaban el programa como alternativa enriquecedora frente a la jubilación.

Por último, a pesar de que para la mayoría de los alumnos la participación en el programa no parecía haber supuesto un gran beneficio a nivel físico, es necesario mencionar que la moda del ítem "He experimentado una mejora en mi estado físico general» fue de 4, con lo que, para muchos, sí ha supuesto un resultado importante.

Es destacable asimismo la baja frecuencia con la que los alumnos indicaron experimentar consecuencias negativas como resultado de su participación en el programa.

Conjuntamente, los resultados mostraron una alta coherencia entre los resultados obtenidos y los resultados percibidos como más importantes (independientemente de si los hubieran conseguido o no). Los alumnos destacaron los resultados a nivel educativocultural, la satisfacción personal por realizar actividades que les enriquecen y la oportunidad que les brinda el programa para ganar relaciones de amistad.

Por otra parte, los alumnos destacaron como relevantes algunos beneficios a nivel psicológico. Los alumnos, aunque afirmaban que obtenían resultados moderados a este nivel, percibían como relevante el impacto que el programa puede tener en este aspecto.

Estos resultados ponen de manifiesto la eficacia del programa educativo de la Universidad de la Experiencia de la Universidad de La Rioja en la consecución de los objetivos previstos en el mismo. En concreto, el programa parece responder a las demandas de formación global de los adultos, fomenta la participación de los mayores en la sociedad, impulsa sus capacidades cognitivas y emocionales $\mathrm{y}$, con ello, mejora la calidad de vida de las personas.

Por otra parte, los resultados obtenidos del análisis del impacto del género son coherentes con estudios anteriores (p. ej., Castellón et al., 2004), indicando ausencia de diferencias estadísticamente significativas, aunque el presente estudio mostró una tendencia general por parte de las mujeres a valorar de manera más positiva que los varones los efectos del programa, especialmente a nivel social y afectivo. Los resultados mostraron que las mujeres en general parecen percibir un mayor impacto a casi todos los niveles de su paso por la Universidad de la Experiencia de la Universidad de La Rioja. Estos datos sugieren que, además de la habitual predominancia del género femenino en este tipo de estudios tanto a nivel nacional (p. ej., Alfageme, 2007) como internacional (p. ej., Davey, 2002), las mujeres son las que más resultados positivos perciben de estos.

Sin embargo, ni la edad, ni la situación laboral ni el nivel de estudios previos parecen tener ningún impacto en la manera en la que los alumnos percibían su paso por el programa.

Estos datos corroboran la consecución de los objetivos del programa independientemente del perfil del alumno, indicando que una de las características más importantes de su diseño, la flexibilidad, se ha materializado y es un proyecto capaz de dar respuesta a las necesidades de las personas, independientemente de su edad, situación laboral y nivel educativo.

Los resultados observados en el presente estudio son coherentes con otros de naturaleza similar (Cabedo, 2010; Castellón et al., 2004; Jamieson, 2007; Montoro y Pinazo, 2005; Rubio Herrera, 2000), que han mostrado altos índices de satisfacción y consecución de objetivos de las personas implicadas en programas universitarios para mayores. La literatura muestra que los estudios realizados con el objetivo de conocer las motivaciones y expectativas de los alumnos para implicarse en programas universitarios para mayores a nivel nacional e internacional (p. ej., Daniel, Templin y Shearon, 1977) han revelado que los principales motivos son mejorar la cultura, sentirse activos, hacer algo nuevo, mejorar la calidad de vida y conocer gente nueva. Los resultados del presente estudio indican que los alumnos del programa universitario de la Universidad de la
Experiencia podrían estar cubriendo estas expectativas de manera muy adecuada. El programa parece satisfacer la curiosidad intelectual y el interés por la cultura de sus alumnos, y promover la integración social, fomentando así niveles superiores de calidad de vida. En este sentido, el presente estudio apoya la tesis de algunos autores (p. ej., Cusak, 2000; Orte y March, 2007) sobre el valor terapéutico y el fomento de la calidad de vida que el aprendizaje aporta a las personas mayores.

Más allá de los beneficios individuales, los resultados del estudio indican la existencia de beneficios sociales que trascienden a los sujetos que participan en el mismo. Los resultados expuestos afectan de manera indirecta a las personas más próximas a los estudiantes, que disfrutan de las consecuencias del mejor estado personal de aquellos, y a la sociedad en su conjunto que se beneficia de un aumento de su capital humano.

Por último, es importante señalar que una de las amenazas a la validez de este estudio fue la deseabilidad social por parte de los alumnos. Se temía que dicha deseabilidad les empujara a contestar de manera reiteradamente positiva. Sin embargo, los resultados obtenidos mostraron que los alumnos eran capaces de contestar de manera discriminada en función del aspecto que estuvieran evaluando. Para conocer el efecto real del programa se requieren estudios con medidas objetivas. En este sentido, el presente estudio constituye un análisis inicial de los beneficios que los alumnos experimentan debido a su participación en este programa universitario para mayores. Se requiere, de cara al futuro, un análisis más exhaustivo de las variables analizadas, con instrumentos de medida estandarizados y con grupos de comparación dentro y fuera del programa para conocer con mayor rigor el impacto que tienen estos programas para los adultos.

\section{Conflicto de intereses}

Los autores declaran no tener ningún conflicto de intereses.

\section{Referencias bibliográficas}

Alfageme, A. (2007). The clients and functions of Spanish university programmes for older people: A sociological analysis. Ageing E Society, 27, 343-361.

Cabedo, A. (2010). La educación permanente: la Universidad y las personas mayores. Castelló de la Plana: Publicaciones de la Universitat Jaume I.

Castellón, A., Gómez, M. A. y Martos, A. (2004). Análisis de la satisfacción en los mayores de la Universidad de Granada. Revista Multidisciplinar de Gerontología, 14, 252-257.

Comisión Europea (2010). Lisbon European Council 23 and 24 March 2000. Presidency conclusions. [consultado 20 Oct 2014]. Disponible en: http://www.europarl.europa.eu/summits/lis1_en.htm

Cusak, S. (2000). Critical educational gerontology and the imperative to empower. Education and Ageing, 14, 21-37.

Daniel, D., Templin, R. y Shearon, R. (1977). The value orientations of older adults toward education. Educational Gerontology, 2, 33-42.

Davey, J. A. (2002). Active ageing and education in mid and later life. Ageing E'Society, $22,95-113$.

Jamieson, A. (2007). Higher education study in later life: What is the point? Ageing E' Society, 27, 363-384.

Marco Español de Cualificaciones (MECU, 2013). [consultado 20 Oct 2014]. Disponible en: http://www.mecd.gob.es/educacion-mecd/areas-educacion/sistemaeducativo/ensenanzas/aprendizaje-largo-vida.html

Ministerio de Sanidad, Política Social e Igualdad. (2010). Libro blanco del envejecimiento activo. Madrid: Instituto de Mayores y Servicios Sociales (IMSERSO). [consultado 20 Oct 2014]. Disponible en: http://www.imserso.gob.es/Inter Present1/groups/imserso/documents/binario/8088_8089libroblancoenv.pdf

Montoro, J. y Pinazo, S. (2005). Evaluating social integration and psychological outcomes for older adults enrolled at a University Intergenerational Program. Journal of Intergenerational Relationships, 3, 65-81.

Navarro, M.C., y Pérez de Albéniz, A. (2010). Los programas universitarios para mayores: la Universidad de la Experiencia de la Universidad de la Rioja. En: Giró J., coordinador. Envejecimiento, conocimiento y experiencia (pp. 109-125). Logroño: Universidad de La Rioja.

Organización Internacional del Trabajo (OIT, 2005). Recomendación 195: Recomendación sobre el desarrollo de los recursos humanos: educación, formación y aprendizaje permanente. Disponible en: http://www.oei.es/pdfs/rec195.pdf

Organización Mundial de la Salud. (2002). Envejecimiento activo: un marco político. Revista Española de Geriatría y Gerontología, 37, 74-105 
Orte, C. y March, M. X. (2007). Envejecimiento, educación y calidad de vida: la construcción de una gerontología educativa. Revista Española de Pedagogía, 237, 347-413.

Orte, C., Ballester, L. y Touza, C. (2004). University programs for senios in Spain: Analysis and perspectives. Educational Gerontology, 30 , 315-328.
Palmero, C. y Eguizábal, A. (2008). Quality of university programs for older people in Spain: innovations, tendencies, and ethics in European higher education. Educational Gerontology, 34, 328-354.

Rubio Herrera, R. (2000). Una aproximación teórica a la experiencia de las Aulas de Mayores. In Rubio Herrera R. (Ed.), Temas de Gerontología III (pp. 324-443). Granada: Grupo Editorial Universitario. 